\title{
Vom kritischen Umgang mit Codes und Medizin
}

\section{On the Critical Use of Codes and Medicine}

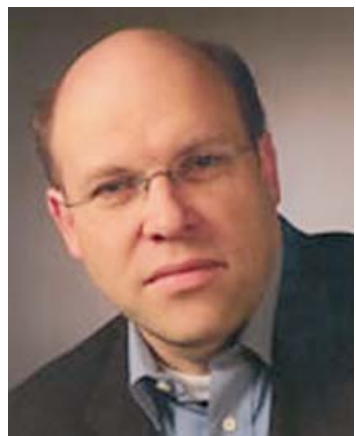

Dr. Matthias Herbst
Die „Aktuelle Dermatologie“ berichtet über die neuesten praxisrelevanten Erkenntnisse aus der dermatologischen Forschung. Nun gibt es Instrumente, die diese Erkenntnisse in der Praxis abbilden: zuallererst einmal die Diagnosen. Im PKV-Bereich ist dies meist zu schaffen, idealerweise passen Diagnose und Therapie zusammen und eine zeitraubende Nachfrage seitens der PKV bzw. des Versicherten bleibt aus. In letzter Zeit häufen sich allerdings Nachfragen seitens der Versicherer insbesondere bezüglich operativer Prozeduren und Dokumentationen. Schreibkram passt nicht zu praxisrelevanter Effizienz und umso öfter hat man den Eindruck, dass man den Arzt durch Bürokratie in eine preiswertere Kategorie der GOÄ-Abrechnung zwingen will.

Im EBM-Bereich droht am Horizont eine zeitintensive Codierwelle. Nun soll die Diagnose zum Code passen und „plausibel“ sein, sonst gibt es kein Geld. Beschäftigungstherapie für zu viele gelangweilte bzw. aufmüpfige Ärzte? Oder vielmehr Arbeitsplatzgarantie für KV-Angestellte? Zunächst einmal muss der Code stimmen und eindeutig sein. Doch da gibt es reihenweise Definitionsprobleme. Betrachten wir die Diagnosestatistik der KV Nordrhein, so fällt uns auf, dass verschiedene Codes z. B. für die aktinische Keratose geführt werden. Nun gibt es solitäre Herde von aktinischen Keratosen und auch flächenhafte Manifestationen auf lichtgeschädigter Haut. Bilden die Codes das ab? Immerhin nimmt die Menge der AK egal unter welcher Codierung in den letzten Jahren deutlich zu.

Warum erfasst die KBV nicht selbst alle Diagnosen im Volltext und baut dann daraus transparent für alle fachlich plausible Gruppen auf, die die Leistung abbilden? Dank bestehender EDV wäre das kein Problem. Die Code-getriggerte Tendenz zu immer subtileren Diagnosen in optimaler Kombination à la Klinik-DRGs bringt ja letztendlich niemanden weiter. Ein ganzes Volk wird offensichtlich sinnlos diagnostisch-statistisch erfasst. Dass 100 Herzinfarkte teurer und mehr wiegen wie 100 Basaliome ahnt jeder. Mit Warzen und Fußpilz in Massen allein können die niedergelassenen Dermatologen das Spiel nicht gewinnen ...

Eine Meldung ans Krebsregister wird durch die Kassenärztlichen Vereinigungen nicht vorgenommen. Hier könnte sinnvoll vereinfacht werden. Durch die Geolokalisation der Diagnosen könnte eine bessere Versorgung erreicht werden: So ließen sich potenziell gesundheitsgefährdende Standorte herausarbeiten und auch die medizinische Versorgung in der Fläche optimieren. Wie gesagt - alles ohne zusätzliches Codieren in der Praxis.

Das Wichtigste fehlt noch: Nur die Regierung und gegebenenfalls die KBV glauben, dass ein Sozialgesetzbuch wirksam vor bösen Buben wie Hackern und Diensten schützt. Spätestens die Online-Übermittlung der Abrechnung von am Internet hängenden Praxis-PCs öffnet dem Diebstahl hochsensibler Patientendaten Tür und Tor. Entsprechende erschreckende Beispiele in sicherheitsrelevanten und daher gut geschützten Einrichtungen wie BKA und auch AKWs gab es in letzter Zeit auffällig viele. Arztpraxen am Netz bemerken Fremdeindringlinge noch nicht einmal. Online-Abrechnung als grobe institutionelle Fahrlässigkeit?!

Eine Empfehlung an die Verantwortlichen zum Schluss: die Diagnosen maschinenlesbar ohne Patientenbezug im Volltext an eine Institution der Versorgungsforschung liefern, um eine wissenschaftlich begründbare Weiterentwicklung der regionalen Versorgung zu erreichen. Der Patient sollte generell der Empfänger der Abrechnung sein, ein Selbstbehalt zur Kostendämpfung die Regel. Nebenbei Praxisgebühr ade!

Medizin für Menschen eben!

Viel Spaß jetzt bei der Lektüre dieses Heftes! Matthias Herbst

\author{
Bibliografie \\ Dol http://dx.doi.org/ \\ 10.1055/s-0030-1256577 \\ Akt Dermatol 2011; 37; 193 \\ (c) Georg Thieme Verlag KG . \\ Stuttgart · New York \\ ISSN 0340-2541
}

Korrespondenzadresse

Dr. Matthias Herbst

Generalsekretär ADK e.V. Bergstraße 1

69120 Heidelberg

Dr.med. Matthias.Herbst@

t-online.de 